\title{
New progress and challenge in gynecological cancer
}

Gynecological cancer seriously threated women's life and health. Cervical cancer, endometrial cancer and ovarian cancer are the most common gynecological cancer, which are all in the top ten common female cancer. According to the latest data in 2020 , the newly diagnosed patients of cervical cancer, endometrial cancer and ovarian cancer respectively have about 600,000 cases, 420,000 cases and 310,000 cases, as accounting for nearly $14.4 \%$ of all new female cancer cases worldwide (1). Cervical cancer is ranking fourth in terms of both morbidity and mortality.

With the wide application of minimally invasive surgery technology, the rapid development of imaging diagnostic technology and also the arrival of the era of big data, we have entered a new stage of precision diagnosis and treatment of gynecological cancer. I am delighted to be the editor for this special series of the Annals of Translational Medicine, titled "New progress and challenge in gynecological cancer". The current special series provides the latest information on biological data, clinical research and translational medicine in the field of gynecological cancer, focusing on new progress and challenge.

In October 2018, the International Federation of Gynecology and Obstetrics (FIGO) terminated the era of clinical staging and introduced postoperative pathological staging for cervical cancer. Imaging diagnosis has been proposed for staging for the first time, leading to significant changes in the diagnosis and treatment of cervical cancer, as well as higher requirements for the sensitivity and specificity of imaging diagnosis. In this series, Liu et al. (2) compared the diagnostic efficacy of Diffusionweighted imaging (DWI) in preoperative staging of cervical cancer with that of magnetic resonance imaging (MRI), and further highlight the correlation between apparent diffusion coefficient (ADC) and clinicopathological characteristics. It is exciting to find that DWI is a useful tool for preoperative evaluation of cervical cancer. ADCmean of local cervical lesions can be more effectively used to predict the need for postoperative complementary therapy, thus playing an important role in assisting clinical treatment decision making. Also, another important significance is that DWI makes the imaging diagnosis of lymph node metastasis more specific. According to the new FIGO 2018 cervical cancer stage, lymph node metastasis will be included in stage IIIC, and it is controversial whether stage IIIC has worse prognosis than stage IIIA and stage IIIB. However, Li et al. (3) conducted a real-world retrospective study of cervical cancer patients from 47 hospitals to discuss the rationality of treatment and tumor outcomes of FIGO 2018 new stage IIIC cervical cancer. The results showed that the outcomes for FIGO 2018 stage IIIC cervical cancer was better than stage IIIB and similar to stage IIIA. Poor prognosis was mainly related to local tumor factors, suggesting that the new FIGO 2018 stage is still worth precise discussion. Meanwhile, in 2018, the results of the Laparoscopic Approach to Cervical Cancer (LACC) trial and the Real World Study (RWS) showed that minimally invasive surgery was associated with higher recurrence and mortality rates, which caused significant concern in the academic community. Serious concerns were raised over the efficacy of minimally invasive surgery for cervical cancer, and the main controversy is that the concept of minimally invasive surgery has been questioned. Using a single-center retrospective case series study, Wang et al. (4) investigated the survival outcomes of abdominal radical hysterectomy (ARH), laparoscopic radical hysterectomy (LRH), and vaginal-assisted laparoscopic radical hysterectomy (VALRH) for cervical cancer. Their results showed that LRH was an independent risk factor for recurrence. VALRH avoided intraperitoneal tumor exposure and achieved the same tumor prognosis as ARH. They also conducted sensitivity analysis for different operators and reached a consistent conclusion, even after excluding the influence of the surgical experience of different operators. This means that VALRH can solve the problem of tumor exposure existing in laparoscopic surgery and lay a foundation for further RCT research. It is expected that minimally invasive surgery after standardizing the concept of tumor-free surgery will still benefit cervical cancer patients.

In addition, this current special series includes another three studies that focus on predicting prognosis for cervical cancer. First, Chen et al. (5) successfully developed a robust and powerful nomogram model for predicting the postsurgical 5-year overall survival (OS) and disease-free survival (DFS) probability, depend on a large multicenter study. Second, Yang et al. (6) employed ESTIMATE and CIBERSORT algorithms to analyze cervical cancer tissue samples from TCGA-CC database, and found that ferroptosis-related genes identify tumor immune microenvironment characterization and the prognostic survival in cervical cancer. Third, Li et al. (7) used twelve miRNA-seq and TCGA-CC tissues to build a molecular risk model for the prognosis of cervical cancer based on microRNA expression. All these studies expand our knowledge and provide new strategies for the predicting prognosis for cervical cancer. 
Besides cervical cancer, studies that focus on ovarian cancer and uterine leiomyosarcoma (uLMS) are also included in this series. Liu et al.'s (8) research develops a powerful prognostic alternative splicing (AS) signature for ovarian cancer and provided a deeper understanding of prognostic splicing factor (SF)-AS network regulatory mechanisms, as well as platinumsensitive and cancer immune microenvironments. These results revealed various candidate biomarkers and potential targets for ovarian cancer treatment strategies. Yang et al. (9) analyzed the risk of morcellation in patients who underwent surgery for leiomyoma and had a final pathological diagnosis of uLMS. They found that no survival differences were observed among the initial surgical procedures in stage I patients with occult uLMS, and no survival benefits were also observed between lymphadenectomy and oophorectomy patients. These new data would provide support for clinical surgical approach strategy.

Finally, we would like to express our appreciation for the contributions of all the authors to this special series. They provided readers with the new research and advances in gynecological cancer, as well as insights into existing challenges with unique perspectives and valuable original data. We believe that the articles in this special series will better promote the transformation of gynecological cancer research "from laboratory to clinic", and also trigger new thinking on it.

\section{Acknowledgments}

Funding: None.

\section{Footnote}

Provenance and Peer Review: This article was commissioned by the editorial office, Annals of Translational Medicine for the series "New Progress and Challenge in Gynecological Cancer". The article did not undergo external peer review.

Conflicts of Interest: The author has completed the ICMJE uniform disclosure form (available at https://atm.amegroups. com/article/view/10.21037/atm-2021-45/coif). The series "New Progress and Challenge in Gynecological Cancer" was commissioned by the editorial office without any funding or sponsorship. DW served as the unpaid Guest Editor of the series. The author has no other conflicts of interest to declare.

Ethical Statement: The author is accountable for all aspects of the work in ensuring that questions related to the accuracy or integrity of any part of the work are appropriately investigated and resolved.

Open Access Statement: This is an Open Access article distributed in accordance with the Creative Commons AttributionNonCommercial-NoDerivs 4.0 International License (CC BY-NC-ND 4.0), which permits the non-commercial replication and distribution of the article with the strict proviso that no changes or edits are made and the original work is properly cited (including links to both the formal publication through the relevant DOI and the license). See: https://creativecommons.org/licenses/by-nc$\mathrm{nd} / 4.0 /$.

\section{References}

1. Wild CP, Weiderpass E, Stewart BW, editors (2020). World Cancer Report: Cancer Research for Cancer Prevention. Lyon, France: International Agency for Research on Cancer. Available online: http://publications.iarc.fr/586

2. Liu L, Wang S, Yu T, et al. Value of diffusion-weighted imaging in preoperative evaluation and prediction of postoperative supplementary therapy for patients with cervical cancer. Ann Transl Med 2022;10:120.

3. Li Z, Duan H, Guo J, et al. Discussion on the rationality of FIGO 2018 stage IIIC for cervical cancer with oncological outcomes: a cohort study. Ann Transl Med 2022;10:122.

4. Wang $\mathrm{H}$, Li D, Wang C, et al. Efficacy evaluation of vaginal-assisted laparoscopic radical hysterectomy and laparoscopic radical hysterectomy for cervical cancer: a single-center retrospective case series study. Ann Transl Med 2022;10:124.

5. Chen X, Duan H, Liu P, et al. Development and validation of a prognostic nomogram for 2018 FIGO stages IB1, IB2, and IIA1 cervical cancer: a large multicenter study. Ann Transl Med 2022;10:121. 
6. Yang X, Yin F, Liu Q, et al. Ferroptosisrelated genes identify tumor immune microenvironment characterization for the prediction of prognosis in cervical cancer. Ann Transl Med 2022;10:123.

7. Li J, Liang L, Xiu L, et al. Establishment of a molecular risk model for the prognosis of cervical cancer based on microRNA expression. Ann Transl Med 2022;10:125.

8. Liu Q, Zhang H, Yang X, et al. Systemic characterization of alternative splicing related to prognosis, immune infiltration, and drug sensitivity analysis in ovarian cancer. Ann Transl Med 2022;10:126.

9. Yang J, Yang J, Cao D, et al. Management of stage I occult uterine leiomyosarcoma: effects of morcellation, second-look surgery, and adjuvant treatments on survival. Ann Transl Med 2022;10:127.



Danbo Wang

\section{Danbo Wang}

Department of Gynecology, Cancer Hospital of China Medical University, Liaoning Cancer Hospital and Institute, Shenyang, China.

(Email: wangdanbo@cancerhosp-ln-cmu.com)

Submitted Dec 17, 2021. Accepted for publication Jan 19, 2022. doi: $10.21037 / \mathrm{atm}-2021-45$

View this article at: https://dx.doi.org/10.21037/atm-2021-45

Cite this article as: Wang D. New progress and challenge in gynecological cancer. Ann Transl Med 2022;10(2):119. doi: 10.21037/atm-2021-45 